\title{
The Mediating Effect of Social Support - A Study on the Effect of Social Participation on the Recovery of Trauma in COVID-19 Elderly Patients
}

\author{
Xiaoping Feng ${ }^{1, a}$, Kaixin $\mathrm{Hu}^{2}$, Liyi $\mathrm{Chen}^{3}$, Zeyi $\mathrm{Liu}^{4}$, Xiaoli $\mathrm{Yao}^{5}$ \\ School of Literature, Law and Economics, Wuhan University Of Science And Technology \\ ${ }^{2}$ School of Literature, Law and Economics, Wuhan University Of Science And Technology \\ ${ }^{3}$ School of Literature, Law and Economics, Wuhan University Of Science And Technology \\ ${ }^{4}$ School of Literature, Law and Economics, Wuhan University Of Science And Technology \\ ${ }^{5}$ School of Literature, Law and Economics, Wuhan University Of Science And Technology \\ axiaoping198284@163.com
}

\begin{abstract}
The outbreak of the COVID-19 epidemic has caused more severe trauma, especially psychological trauma, to the physically and psychologically vulnerable elderly patient population. Even after the elderly patients have recovered from the disease, the psychological trauma continues to have a negative impact on both the physical and mental health of the elderly patients, causing them unable to live as usual. In this study, using the case study approach of qualitative research, we conducted semi-structured interviews with two representative and typical cases of elderly patients to develop in-depth knowledge of the process of mental health recovery after physical healing. The study suggests that for promoting the mental health recovery of elderly patients with COVID-19, it is important to improve the level of social support for elderly patients while encouraging them to actively participate in social activities, so that elderly patients can achieve better self-reconstruction and thus be able to actively cope with the negative social feedback they may face during activities participation and improve the effect of social participation on mental health recovery.
\end{abstract}

Keywords COVID-19, mental health recovery, elderly patients, social participation, social support

\section{INTRODUCTION}

In addition to the physical effects of the COVID-19 outbreak, it may also have different degrees of psychological impact on different groups of people. Most patients with COVID-19 suffer from varying degrees of depression, anxiety, insomnia, and other undesirable psychological conditions, and even suffer from more significant psychological trauma after physical healing, which can have a lasting negative impact on their lives. Elder patients, as the most vulnerable group of patients in the COVID-19 patient population, are subject to greater risk and trauma from physical to psychological. According to data released by the CDC, as of June 23, 2021, in the distribution of confirmed cases and deaths of COVID-19 by age group in the United States, the elderly patients aged 65-74 accounted for $7.4 \%$ of the total confirmed case and $21.6 \%$ of the total deaths; $3.9 \%$ of the total number of confirmed cases and $27.4 \%$ of the total number of deaths among older patients aged 75-84. The average morbidity and mortality rate in the United States was
$1.8 \%, 5.3 \%$ for those aged $65-74$ years, and $12.7 \%$ for those aged 75-84 years. ${ }^{[1]}$ On April 2, 2020, Hans Kruger, director of the World Health Organization Regional Office for Europe, stated that more than $95 \%$ of deaths from COVID-19 in the European region were among people over the age of $60 .{ }^{[2]}$ Thus, from the medical point of view, elderly people show more vulnerability in the epidemic due to their own age characteristics, physical state and other factors, which means they need more attention and help from the society. At the same time, from the psychological point of view, like other groups suffering from the disease, in addition to physical damage, elderly patients also suffer from different degrees of psychological trauma. Moreover, due to the weakening of social roles and social functions brought about by the age of the elderly themselves, elderly patients may face greater social difficulties in recovering from psychological trauma, especially when reintegrating into community life after physical healing, they may encounter more resistance than we thought. Meanwhile, as the global aging process accelerated, more and more elderly people are living 
alone, and those recovering from COVID-19 may face the problem of unmet psychosocial support needs, ${ }^{[3]}$ making the trauma recovery of elderly patients even more difficult. Even if they have recovered physically from COVID-19, psychological burdens and trauma that are not well relieved will still seriously affect the physical and mental health and the quality of life of the elderly. Therefore, the seriousness of psychological problems in elderly patients with COVID-19 cannot be ignored, and research and exploration on helping elderly patients with COVID-19 to recover from psychological trauma cannot be delayed.

\section{LITERATURE REVIEW}

There are several perspectives in the existing studies addressing the factors that influence the mental health recovery of elderly patients with COVID-19: at the level of the individual older person: the patient's attitude and coping style towards the disease have an important impact on mental health recovery. Kato (2016) et al. found that good attitudes can lead to a range of successful adaptations to adverse circumstances and can have a protective effects. ${ }^{[4]}$ Shallcross et al. also emphasized that acceptance of negative experiences can protect individuals from negative emotions and prevent depression. ${ }^{[5]}$ The result of an empirical study by Sun Juanjuan and Jiang Weikang showed that both positive coping and negative coping can directly affect the mental health of older adults, but positive coping play a gain role while negative coping weakens the level of mental health. ${ }^{[6]}$ However, all of the above studies only focused on the psychological effects of individual external coping styles of the elderly, without discussing the underlying reasons why different elderly people adopt different coping styles, and ignored the interaction between external factors and individual responses and its effects.

Another part of the existing research looks at the social environment in which the elderly are living: social stigma has a very important role in the recovery of elderly patients with COVID-19. ${ }^{[7]}$ Patients recovering from COVID-19, even after being removed from strict medical isolation, are still in a social situation close to "quarantine isolation" and face a range of problems such as discrimination, difficulty integrating into community life, and active social avoidance. These conditions may lead to various psychological problems such as depression, anxiety, fear, loneliness, resentment, and confusion (Lin et al., 2010). ${ }^{[8]}$ As Colins (2020) recently noted, "If people are deprived of concrete interactions, they will be more likely to become depressed, less energetic, and have a reduced sense of solidarity with others, and therefore become more anxious, distrustful, and sometimes hostile." ${ }^{[9]}$ Thus, it is clear that the element of social distance cannot be ignored when analyzing the physical and mental health recovery of elderly patients with COVID-19, and that creating opportunities and encouraging the elderly to increase their participation in social activities and bring them closer to the society may be an effective way to alleviate the psychological problems of elderly patients with COVID- 19 .

Activity theory has analyzed and explained the relationship between the level of mental health and social activity participation of the elderly. Activity theory encourages the elderly to actively engage in society, and only by participating in society can the elderly rediscover themselves and maintain the vitality of their lives. ${ }^{[10]}$ According to this view, older adults with high activity levels are more likely to feel satisfied with their lives and adapt to society more easily than those with low activity levels. Besides, there are many other analyses in existing studies about the relationship between the mental health level of older adults and social activity participation. For example, some scholars believe that a certain level of social participation can help to enhance the sense of self-worth, improve their mental state and interpersonal skills, as well as meet the need of emotional communication and thus improve the mental health level of the elderly. Related studies have mostly used quantitative analysis methods such as measurement indicators to explore the effects of social participation activities on the psychological health of older adults, further proving the activity theory that older adults should actively participate in social activities. Although the studies prove the activity theory's view that older adults need to be actively involved in social activities, they do not answer the question of what specific mechanisms of social participation affect the level of mental health of older adults. The answer to this question is crucial for a deeper understanding of the relationship between mental health and social activity participation in older adults.

In a study of the relationship between social support and individual mental health, Kawach (2001) ${ }^{[11]}$ found that social support has beneficial effects on the lives and physical and mental health of older adults, and the more social support, the higher the level of mental health. According to $\mathrm{Li}$ (1998), ${ }^{[12]}$ the relationship between social support and individual psychological health is very close and direct, and social support has a very important influence on relieving individual psychological stress, eliminating individual psychological barriers, and enhancing individual psychological health. He cites two hypothetical models on how social support affects individual psychological health. The first is the main effect model, which suggests that social support plays a role in maintaining the individual's usual good emotional experiences and physical and mental conditions; the second is the buffer model, which suggests that social support only plays a 
buffering role in stressful situations to maintain mental health conditions.

In the existing studies, either a direct correlation between social participation and mental health recovery or a direct correlation between social support and mental health recovery has been established. The purpose of this study is to explore, through the analysis of case interview materials, how the level of social support affects the individual's specific social participation behaviors, including the way the individual responds to external feedback, and thus affecting the individual's mental health recovery.

\section{DATA SOURCES AND RESEARCH RESULTS}

\subsection{Data sources and research methods}

This study mainly used the case study method in qualitative research and selected two representative and typical cases of elderly patients with COVID-19 from the community of Qili Yicun in Wuhan city, the site of the outbreak in China. The community of Qili Yicun in Wuhan is a very typical aging community, and most of the community residents are retired workers from factories in the same area, and the neighborhoods are very familiar with each other, and the community reflects a strong bonding. The experiences of two selected cases in this community focus on the social participation dilemmas and trauma recovery processes faced by the group of elderly patients with recovered COVID-19 as they return to community life after treatment and medical isolation.

The researcher collected data mainly through the following ways: literature analysis. The relevant research literature was reviewed and organized to understand the relevant research results about theories and methods of psychological trauma recovery in patients with COVID-19; the gaps in existing research and areas for improvement were collated and analyzed. Semi-structured interview. According to the interview outline, questions were asked to the cases, and they were also encouraged to ask their own questions. In the interview, we learned about the resistance to social participation they face, ways of coping with resistance, and the trauma recovery journey, after their returning to community life.

Table 1. Basic information of the cases

\begin{tabular}{|l|l|l|}
\hline Interviewees & Auntie Tsang & Auntie Liao \\
\hline Age & 71 years old & 64 years old \\
\hline Professional Background & Accountant, retired & Nurse, retired \\
\hline Residence & Living alone & Living with daughter \\
\hline Rehabilitation & Good physical recovery & Good physical recovery \\
\hline Economic Background & $\begin{array}{l}\text { Monthly income pension 3500 } \\
\text { yuan }\end{array}$ & $\begin{array}{l}\text { Monthly income pension 3500 } \\
\text { yuan }\end{array}$ \\
\hline Family members & Widowed with a daughter & Widowed with a daughter \\
\hline
\end{tabular}

\subsection{Study results}

\subsubsection{Dilemma and Action}

The two cases live in the same community, which is mainly populated by retired elderly people. According to Social Role Theory, after individuals retire from their occupational roles, they must have new roles to replace them, and need to be compensated with social activities in order to adapt to their retirement life [13]. The two elderly people in this study were accountant and nurse before retirement, and had a rich retirement life before their illnesses, both of whom were active in various leisure activities in the community, completing the transition from occupational to leisure roles and in good physical and mental health. Moreover, the community of Qili Yicun, where the cases live, is a typical acquaintance community, where neighbors are familiar with each other and social distance is close.
However, after the illness, the high degree of community bonding makes the patients' illness easily known to other residents in the community on the one hand, and on the other hand makes the cases feel a strong change in social distance when returning to the community. The social distance is greatly distanced, causing a huge psychological gap, thus aggravating the dilemma of recovering from the psychological trauma.

Auntie Liao felt the sudden increase of social distance. Previously familiar neighbors have shown reluctance to approach her or talk with her, which made Auntie Liao feel less close to her neighbors.

" Sometimes, especially when I first got sick, I really feel a big difference", " People who didn't know I were sick were still kind to me, and those who knew were embarrassed to act too distant. But in fact, I spoke with them a few words, looking at their performance, then I knew we were not so close as before".

In addition, Auntie Liao also felt the rejection of the former activity groups, so she had to consciously avoid 
such activities, so that the former social role established in the community was weakened or even deprived.

" I used to be quite active, like to engage in various activities for the elderly, but after I got this disease, I was afraid that other people would not want me to join, so I could only visit occasionally and move around from a distance".

Aunt Zeng felt the comments from behind her back, making her feel uncomfortable because she felt that she was not treated as a normal person.

" Those who discussed me behind my back make me very unhappy. When I saw the dentist before, I heard the doctors and nurses whispering about me ...... If they said it to my face, I could accept it, behind my back, I was unhappy and uncomfortable "

In the process of the cases' return to the community after healing, the alienation of neighbors, the backbiting and the rejection of the cases by their former social activity groups increase the social distance between the cases and the community residents[9], and this social dilemma aggravates the psychological dilemma of the cases. On the one hand, the social dilemma of partial disintegration of the social support network and difficulty in reintegrating into community life exposes the cases to mental health hazards such as depression, loneliness, and resentment [8]. On the other hand, social alienation leads to the inability of the cases to participate in community activities, which makes the cases lose their social role. The disintegration of community social support and the loss of social role make the cases' social function weakened [14], a situation that makes it difficult for the cases to be understood by others and accepted by society.

Despite facing both social and psychological difficulties, both cases maintained a positive attitude toward various opportunities for community participation and took positive actions. Activity theory encourages the elderly to actively participate in the community, and only by participating in the community can the elderly reconnect with themselves and maintain the vitality of life [10]. In the process of participating in community activities, the two cases found a sense of belonging and identity among similar groups, which not only alleviated their psychological trauma, but also restored their social interaction and social function to a certain extent, and promoted the individual self-restoration.

"The community was doing okay, the activity definitely got better. In that activity, we participants could play and talk together. They also invited a psychological counselor to lecture twice." (Auntie Liao)

"I went every time, whenever there was this activity. I could make friends and talk, otherwise there's usually no one to talk to", "I'm also willing to do anything the community wants me to do, as long as it's not too tiring, such as sweeping the floor, I'm willing to do it. Just do not know if I am needed." (Auntie Zeng)

In addition to the homogeneous group activities organized in the community specifically for the COVID-19 patients, both cases had an expectant and positive attitude towards more community activities.

\subsubsection{Response and feedback}

Facing the social dilemmas of social distancing and reintegrating into the community, both cases actively participated in community activities, sought and established community connections, and reintegrated into the community. According to the current studies, the above-mentioned social activities are beneficial to the physical and mental health of the elderly, and are helpful in overcoming the social and psychological difficulties of reintegration into the community. However, the interviews found that the two cases had different attitudes and behaviors toward the resistance they encountered in the specific process of social participation.

When Auntie Zeng was seeing her dentist, she heard whispers from the doctors and nurses about her having had COVID-19, she felt psychologically uncomfortable and adopted a negative, avoidant attitude and behavior.

"It's okay when they say it to my face, but when they say it behind my back, I'm not happy and feel uncomfortable with it. I'd rather eat something soft and wait until next year than see the dentist."

Auntie Zeng's way of coping is the "repression" of the ego defense mechanism. "Repression" is regarded as the most basic method of psychological defense mechanism, and is one of the escape mechanisms, which refers to the rejection and prohibition of the ego's wishes, memories, desires, and hobbies by the superego, thus affecting people's behavioral performance[15].During the interview, Auntie Zeng talked about the incident with a grave expression and indignant tone; she felt that the incident was unfair to her and made her feel hurt, and she did not want to be treated in this way. However, in response, Auntie Zeng suppressed her inner emotions and desires, and did not show active expression and self-defense. Such an approach neither transformed nor dealt with the social dilemma of reintegration into the community, but also left the emotions bottled up in her heart without being reasonably resolved or reasonably explained, thus aggravating Auntie Zeng's psychological dilemma and thus negatively impacting the recovery from the trauma.

While Auntie Liao showed positive attitudes and behaviors in the face of social distance alienation and stigmatization, taking the initiative to justify herself and 
responding to social dilemmas through positive explanatory methods.

"People directly said, directly said to you, I am afraid of you, I'm afraid of being with you...... What else can I say, I can only say that I wear a mask well, I also have antibodies, and will not infect other people, you guys needn't be afraid."

According to the American social psychologist William Schutz (1958)[16], every individual has the desire and need for interpersonal interaction, and different people have different needs. Schutz classified interpersonal needs into three categories: inclusion, control and affection, and each of these needs has two different orientations: active orientation and passive orientation. In this study, both Auntie Zeng and Auntie Liao were very eager for inclusion needs (the need to establish and maintain harmonious relationships with others. The active orientation of interpersonal interaction stimulated by this need is to actively interact with others and actively participate in social life, while the passive orientation is to withdraw, isolate, and expect acceptance from others.) and emotional needs (the need to establish and maintain intimate connections with others in feelings. The active orientation of interpersonal interaction motivated by this need is to show friendliness, affection, sympathy and intimacy to others, while the passive orientation is to be indifferent and expect others to show intimacy to oneself.)

During the interview process, it was found that Auntie Zeng tended to be more of a passive orientation, passively expecting understanding, acceptance and closeness from others, while Auntie Liao showed more of an active orientation, taking the initiative to understand, accept and communicate.

"If I had been with people without COVID-19, I wouldn't have been able to say so much..... but now I can say it out. If this activity hadn't happened, we would have had to hold it in our heart.", "I am very willing to do whatever the community asks me to do." (Auntie Zeng).

" But I think this problem will slowly die out over time, it will be better, right? It is also understandable, think about it, if it were me, I would mind more or less, right? It is normal", "So make it clear to them, that if he still doesn't get it, there's nothing you can do. Just to save face, then wear a mask and talk as little as possible." (Auntie Liao).

\subsubsection{Support and reconstruction}

Both cases hold a proactive attitude toward participation in community activities and give positive actions. Among the available research findings, scholars generally agree that encouraging greater participation in community activities for groups of older adults who have been treated with social distance will help them improve their physical and mental health and better reintegrate into the community.

However, by interviewing two typical cases, researchers found that although they were both actively involved in community activities, they responded very differently to the negative feedback from the community in specific social engagement activities, and the different responses further influenced the recovery of mental health in the cases. The extent to which social engagement activities improve mental health is also influenced by the way individuals respond to external feedback in specific activities. Since there is little discussion of the underlying causes of the different coping styles in existing research, researchers sought to investigate the factors that influenced the different coping styles of the two cases in the face of negative feedback from the society.

After further in-depth interviews, we found significant differences between the two cases in terms of social support networks.

Auntie Zeng, who has a more negative and passive coping attitude, is currently living alone, her partner passed away many years ago, her daughter visits occasionally, and she was an accountant at school before she retired. Before her illness, Auntie Zeng's social support network consisted mainly of her daughter, colleagues at school and neighbors in the community. After her illness, Auntie Zeng voluntarily quit her job as an accountant, and her neighbors in the community also distanced themselves from Auntie Zeng because of her illness, so Auntie Zeng's social support network became very weak.

Because of this disease, everyone is estranged and I can't do school work anymore. As I am getting older and suffering from this disease, my neighbors just said hello from afar when they saw me. My daughter is also busy. When I was just discharged from the hospital, she didn't dare to come in my home when she came to see me, only putting the things she brought to me at the door.", "Thank you (referring to the researchers), really Thank you, come to talk with me " (Aunt Zeng).

In contrast, Auntie Liao's social support network is much stronger. She lives with her daughter and worked as a nurse before retiring, and she is still invited to relatives' gatherings after she has recovered from her illness.

"During the Spring Festival, I still visited my relatives and friends. Tomorrow they said they would take me to see one of my elders because they were afraid that I would miss them, so we could finally get together. I'm still thinking about not being all around together. Eating is also inconvenient. Although they persuaded me it's okay, and we use public chopsticks, I 
guess that people still have some opinions in their hearts.", "Fortunately, with my daughter, several other relatives and friends took turns to visit me. Although I said no need to take care of it, in fact, no matter whether it is taken care of or not, it's still different if someone talks to yourself." (Aunt Liao).

The buffer model of social support suggests [12] that social support often works through a person's internal cognitive system. Social support can play a considerable role in the middle link of the chain of the relationship between stressful events and health status. Social support influences the intermediate link between stressful events and subjective evaluations. Compared with Auntie Zeng, Auntie Liao received stronger social support, and her friends and familyadvised her that she was "fine", which helped her rebuild her perceptions to some extent. As a result, due to the relatively good cognitive reconstruction, Auntie Liao's estimation of the harmfulness of the stressful situation was relatively small, so she would adopt a more proactive attitude and adopt a relatively relaxed psychological state to cope with the negative social feedback she faced when she returned to the community.

According to a related empirical study [17], social support showed a positive relationship with self-efficacy level. Auntie Liao, who had better social support, showed higher self-efficacy levels when coping with stressful situations, and she believed she was capable and better able to cope with negative social feedback, thus Auntie Liao would be willing to take a more proactive coping approach compared to Auntie Zeng's negativity and avoidance.

The ability of an individual to adapt well in the face of stress and distress is a positive psychological state, which is defined as "psychological resilience" by the American Psychological Association. In an empirical study of community-based hypertensive patients [18], Li et al. found that social support influenced psychological resilience through the mediator of self-efficacy.

Therefore, Auntie Liao's good social support can help rebuild an individual's cognition and enhance self-efficacy level, which in turn enables Auntie Liao to show good adaptability and have better "psychological resilience". Based on this better self-reconstruction, Auntie Liao would take a proactive approach in the face of stressful situations, thus promoting the recovery of mental health. Aunt Zeng's relatively weak social support could not help her to complete this process.

\section{DISCUSSION}

For the pathway to help elderly patients with COVID-19 improve their mental health recovery, the activity theory in existing studies advocates that by encouraging the elderly to actively participate in social activities, thereby enhancing life vitality and improving mental health. In this study, through survey interviews with two typical cases, it was found that the theory does have practical implications, and the activity participation in both cases promoted their social interactions and helped their social functions to continue, enhanced their self-identity to a certain extent, and thus promoted their psychological health.

However, the researcher argues that the existing studies do not adequately discuss the specific mechanisms by which activity theory occurs and whether there are other influencing factors, and that further research should be conducted on the specific social activity participation process. The main component of social activity participation in both cases was a proactive return to the community and reintegration into community life, however, negative feedback from the community was received during this activity process. Those who were able to respond with positive attitudes showed higher levels of mental health recovery, and those who responded with negative attitudes showed lower levels of mental health recovery. It is thus clear that the extent to which social activity participation in activity theory contributes to patients' mental health recovery is also influenced by the way in which cases respond to negative social feedback during specific activities.

After further in-depth interviews with the cases, the researcher found that the differences in coping styles were fundamentally due to differences in the strength of social support in the cases. While existing studies have discussed the relationship between social support and self-efficacy, psychological resilience, and psychological well-being, in this study, the researcher found that social support was also closely related to the way individuals responded to negative feedback. The case with stronger social support networks showed higher level of self-reconstruction by having more self-identification, a relatively relaxed state of mind when coping with stressful situations, and more self-confidence. The high level of self-reconstruction not only keeps the individual in a better state, but also makes the individual more resilient when facing negative stimuli such as negative social feedback, thus promoting the individual to take a proactive approach to cope and resolve the difficulties faced, and the process of proactive coping itself is a kind of reinforcement of self-reconstruction, which in turn has a direct effect on the individual's mental health recovery level. The process of positive coping itself is also a kind of reinforcement of self-restoration, which in turn has a direct effect on the level of mental health recovery. 


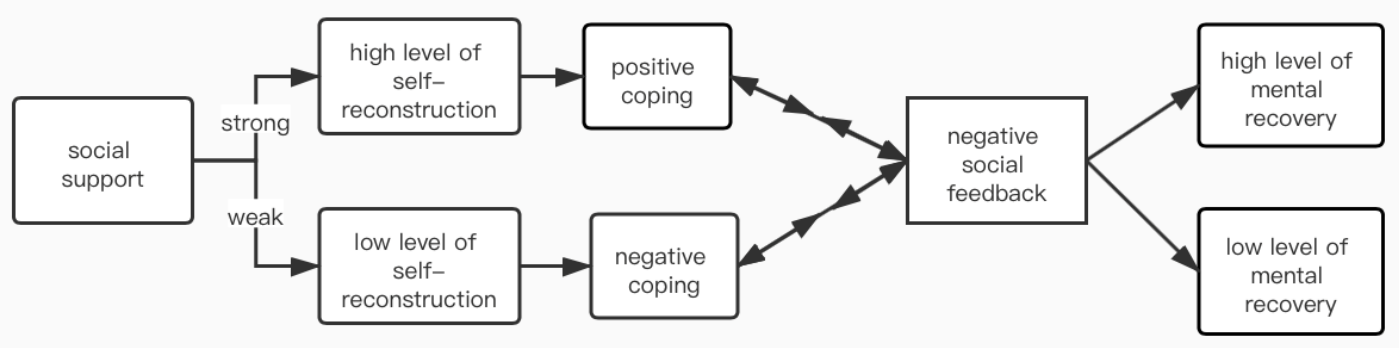

Figure1 Social Support - Coping Style - Mental Recovery Model

\section{CONCLUSION}

In summary, the researchers concluded that the strength of social support affects the level of individual self-reconstruction in individuals with COVID-19, controlling for variables such as gender, level of social participation, and community environment. In turn, different levels of individual self-reconstruction will lead to positive, active or negative, passive coping styles when individuals engage in social activities and face negative feedback from society such as stigmatization and social alienation. Positive coping styles promote the resolution of social dilemmas and provide reinforcement for self-reconstruction, thus allowing individuals to better utilize the role of activity participation in promoting mental health levels during social activity participation. Negative coping styles, on the other hand, do not achieve such effects. Therefore, for helping elderly patients with COVID-19 to recover their psychological health, it is necessary not only to encourage active participation in social activities, but also to focus on enhancing patients' social support networks.

However, this study is still flawed in its discussion of what two-way responses occur during the specific process of interacting with different coping styles and negative social feedback, and what other factors affecting mental health may be triggered during that two-way interaction, which needs to be studied in more depth and detail.

\section{ACKNOWLEDGEMENTS}

On the completion of my dissertation, I would like to thank my supervisor, Ms. Feng Xiaoping. From the selection of the thesis, the writing of the body to the final repeated revisions, Ms. Feng has taken the trouble to provide detailed annotations and valuable comments. I would also like to thank the staff of Wuhan Qili Yicun community for their enthusiastic help in interview liaison and data sharing. Finally, I would also like to thank all the people who worked together to fight the epidemic, and I believe that through our efforts, we will eventually overcome the epidemic.

\section{FUNDS}

Survey on the Current Situation of Social Work Agencies in Hubei Province and Analysis of Countermeasures (HBNPO202005YB)

Hubei University Student Innovation and Entrepreneurship Training Program (S202010488007)

Hubei Province Undergraduate Training Program for Innovation and Entrepreneurship

\section{REFERENCES}

[1] Demographic Trends of COVID-19 cases and deaths in the US reported to CDC Centers for Disease Control and Prevention website.https://COVID.cdc.gov/COVID-data-tra cker/\#demographics

[2] GENEVA, April 2 (RIA Novosti) - More than 95 percent of deaths from neocoronary pneumonia in the European region are among people over 60 years of age.https://sptnkne.ws/GD4B

[3] United Nations (2020). Policy brief: The impact of COVID-19 on older persons.

[4] Kato, K., Zweig, R., Schechter, C. B., Barzilai, N., \& Atzmon, G. (2016). Positive attitude toward life, emotional expression, self-rated health, and depressive symptoms among centenarians and near-centenarians. Aging \& amp; Mental Health, 20(9), 930-939.

[5] Shallcross, A. J., Troy, A. S., Boland, M., \& Mauss, I. B. (2010). Let it be: accepting negative emotional experiences predicts decreased negative affect and depressive symptoms. Behaviour Research and Therapy, 48(9) ), 921-929.

[6] Sun, Juanjuan, Jiang, Weikang. Negative life events and the mental health status of Chinese older adults [J]. Population Research, 2020, 44(2):73-86.

[7] Ramaci, T., Barattucci, M., Ledda, C., \& Rapisarda, V. (2020). Social Stigma during COVID-19 and its impact on HCWs outcomes. sustainability, 12(9), 3834 
[8] Lin, E. C. L., Peng, Y. C., \& Hung Tsai, J. C. (2010). Lessons learned from the anti-SARS quarantine experience in a hospital-based fever screening station in Taiwan. American Journal of Infection Control , 38(4), 302-307.

[9] Randall Collins.(2020). Social distancing as a critical teat of the micro-sociology of solidarity. American Journal of Cultural Sociology(2020), $8: 477-497$.

[10] Zhang Sayu. A peek at the current situation of "lost" elderly people and countermeasures from the perspective of activity theory[J]. Journal of Social Sciences of Shanxi Higher Education Institution, 2016(7).

[11]Kawach I, Berkman L F. Social ties and mental health [J].Journal of Urban Health, 2001, 78(3):458-467.

[12] Li Qiang. Social support and individual psychological health [J]. Tianjin Social Science, 1998(1):66-69.

[13] Jiang De-zhen. Changes in the social roles of the elderly and their adjustment[J]. Journal of PLA Health Care Medicine,2002,4(3):189-190.
[14] Blazer, D. G. (2000). Psychiatry and the oldest old. The American Journal of Psychiatry, 157, 1915$-1924$.

[15] (US) Blackman, Guo Daohuan et al. The Mask of the Mind: 101 Psychological Defenses: East China Normal University Press, 2011

[16] Schtuz WC.FIRO: A three-dimensional theory of interpersonal behavior [M].New York: Holt Rinehart Winston,1958:1.

[17] Luan W, Zhu Z, Zhu D P, Ge W J, Zhu Qun M, Jiang Y Q, Li J, Jiang A L. A study on the correlation between health promotion lifestyle and social support and self-efficacy among elderly people in urban-rural integrated communities[J]. Journal of Shanghai Jiaotong University (Medical Edition),2020,40(01):107-111.

[18] Li C-M, Zhang H-M, Gao J, Zhang R-Q, Wang H, $\mathrm{Yu} \mathrm{H}-\mathrm{Z}$. The relationship between social support and psychological resilience in community hypertensive patients: an analysis of the mediating effect of self-efficacy $[\mathrm{J}]$. Chinese Journal of Gerontology,2021,41(07):1507-1511. 